\title{
CRECIMIENTO JUVENIL DE 32 PROCEDENCIAS Y 203 FAMILIAS DE Eucalyptus globulus ssp.globulus EN LA ZONA COSTERA DE LA VIII REGION DE CHILE
}

\author{
Pedro Infante L. $\left(^{*}\right)$ \\ José Antonio Prado D. (*)
}

\section{RESUMEN}

La supervivencia y desarrollo de 32 procedencias y 203 familias de Eucalyptus globulus ssp. globulus, que cubren gran parte de la distribución natural de la especie, se evalua en un ensayo de procedencias y progenies establecidoen la provincia de Arauco, Chile.

Veinte meses después de la plantación se encuentran diferencias significativas entre procedencias y familias, tanto en altura como en diámetro.

Aún cuando no hay un patrón de variación geográfica bien definido, estos resultados indican que las mejores procedencias corresponden al sudeste de Tasmania, las islas Cape Barren y Flinders, en el Estrecho de Bass y Otway, en Victoria.

La fuente de semillas local fue superada por 15 procedencias naturales.

Palabras claves: Eucalyptus globulus. Procedencias. Progenies.

\section{ABSTRACT}

Survival and growth of 32 provenances and 203 families of Eucalyptus globulus ssp. globulus from almost the whole range of the natural ocurrence of the species, where assessed in a progeny test established in Arauco province, Chile.

Twenty months after planting there was significant variation among provenances and families, both in height and diameter.

Eventhough there is not a well defined pattern of geographical variation, these first results indicate that the best provenances are those from south east Tasmania; Cape Barren Island and Flinders Island in Bass Strait; and Otway, Victoria.

The local seed source was outperformed by 15 natural provenances.

Keywords: Eucalyptus globulus. Provenances. Progeny. 


\section{INTRODUCCION}

La creciente demanda mundial por productos forestales y particularmente por madera de fibra corta para la industria de pulpa y papel, ha despertado un gran interés por plantar especies del género Eucalyptus, tanto en Chile como en otros paises del mundo.

En Chile gran parte de la producción de materia prima y de productos elaborados está destinada a ser vendida en el mercado externo, donde la competitividad es un factor fundamental. Mantener esta posición en el mercado significa producir al mínimo costo, proceso que se inicia obteniendo el máximo de productividad de los suelos forestales. Para esto es necesario aplicar técnicas intensivas de establecimiento, una adecuada selección de la especie a emplear y un proceso de mejoramiento genético que permita lograr la máxima productividad que su base genética pueda entregar.

Con este objetivo, el Instituto Forestal está desarrollando un programa de mejoramiento genético de varias especies del género Eucalyptus, entre ellas Eucalyptus globulus ssp. globulus, una de las especies de mayor importancia en Chile, por su adaptación a sitios muy diversos, por su gran capacidad de retoñación, que la hace adecuada para un manejo en rotaciones cortas, y especialmente por la alta calidad de su madera para la producción de pulpa.

Eucalyptus globulus ssp. globulus es una especie originaria de Australia, específicamente de los Estados de Victoria y Tasmania, incluyendo las islas del Estrecho de Bass donde se la encuentra formando bosques mixtos con varias especies, entre las que se pueden mencionar Eucalyptus viminalis, Eucalyptus obliqua, Eucalyptus delegatensis y Eucalyptus regnans (Boland et al. 1984).

Existen además tres especies estrechamente relacionadas, por lo que Kirkpatrick (1975) las agrupa en una especie con cuatro subespecies. El grupo está constituido por E. globulus ssp. globulus, E. globulus ssp. bicostata, E. globulus ssp. maidenii y E. globulus ssp. pseudoglobulus.

Aún cuando la distribución de la especie no es muy amplia, en comparación a otras especies del género, dentro de su rango de ocurrencia natural se pueden encontrar condiciones muy variadas, dando origen a procedencias de semillas que pueden producir grandes diferencias, tanto en el desarrollo de los árboles (Volker and Orme. 1988) como en la calidad de la materia prima (Turner et al., 1983).

A esta variación producida por condiciones de sitio diferentes, se agrega la variabilidad que existe entre árboles de una misma especie aún creciendo en condiciones semejantes. 
Gran parte de esta variabilidad se reúne en los ensayos de procedencias y progenies de E. globulus ssp. globulus que ha establecido el Instituto Forestal. La intensiva selección a que esta población será sometida en los próximos años permite esperar una importante ganancia genética en características tales como crecimiento, densidad de la madera, rendimiento en pulpaje, resistencia al frío, resistencia a plagas y enfermedades $u$ otras características que pueden ser de importancia a futuro.

El presente trabajo constituye una primera indicación en cuanto a las posibilidades de mejoramiento que ofrece la base genética existente. Al comparar el desarrollo de varias familias con la población local, representada por semilla comercial obtenida en el Centro de Semillas de Chillán, ya se visualiza una importante ganancia en crecimiento, que en un mediano plazo puede ser incorporada a las plantaciones industriales que se efectúen.

\section{OBJETIVO}

El presente trabajo tiene como objetivo entregar las primeras indicaciones en relación a las procedencias más adecuadas para la plantación de Eucalyptus globulus ssp. globulus en la zona costera central de Chile, a través del análisis de uno de los ensayos de procedencia y progenie que ha establecido el Instituto Forestal como parte de su programa de mejoramiento genético de varias especies del género Eucalyptus.

\section{MATERIAL Y METODO}

\section{Antecedentes de la Colección de Semillas}

Eucalyptus globulus ssp. globulus es originario de Australia, específicamente de la zona sur del Estado de Victoria, de las Islas del Estrecho de Bass y Tasmania (Figura № 1).

La colección de semillas empleada en el ensayo es una muestra que representa parte importante del área de distribución natural de la especie (Cuadro $\mathrm{N}^{\circ} 1$ y 
Figura $\mathrm{N}^{\circ} 1$ ). Las semillas se encuentran individualizadas según lugar de origen (procedencia) y arbol madre (familia). La colección incluye 32 procedencias y 203 familias australianas, a las que se agregaron como testigos dos procedencias nacionales (Talca y Cañete), una muestra de huerto semillero (APPM, Tasmania, Australia), una procedencia de Eucalyptus globulus ssp. bicostata, una procedencia de E. globulus ssp. maidenii y una familia de E. globulus ssp. pseudoglobulus.

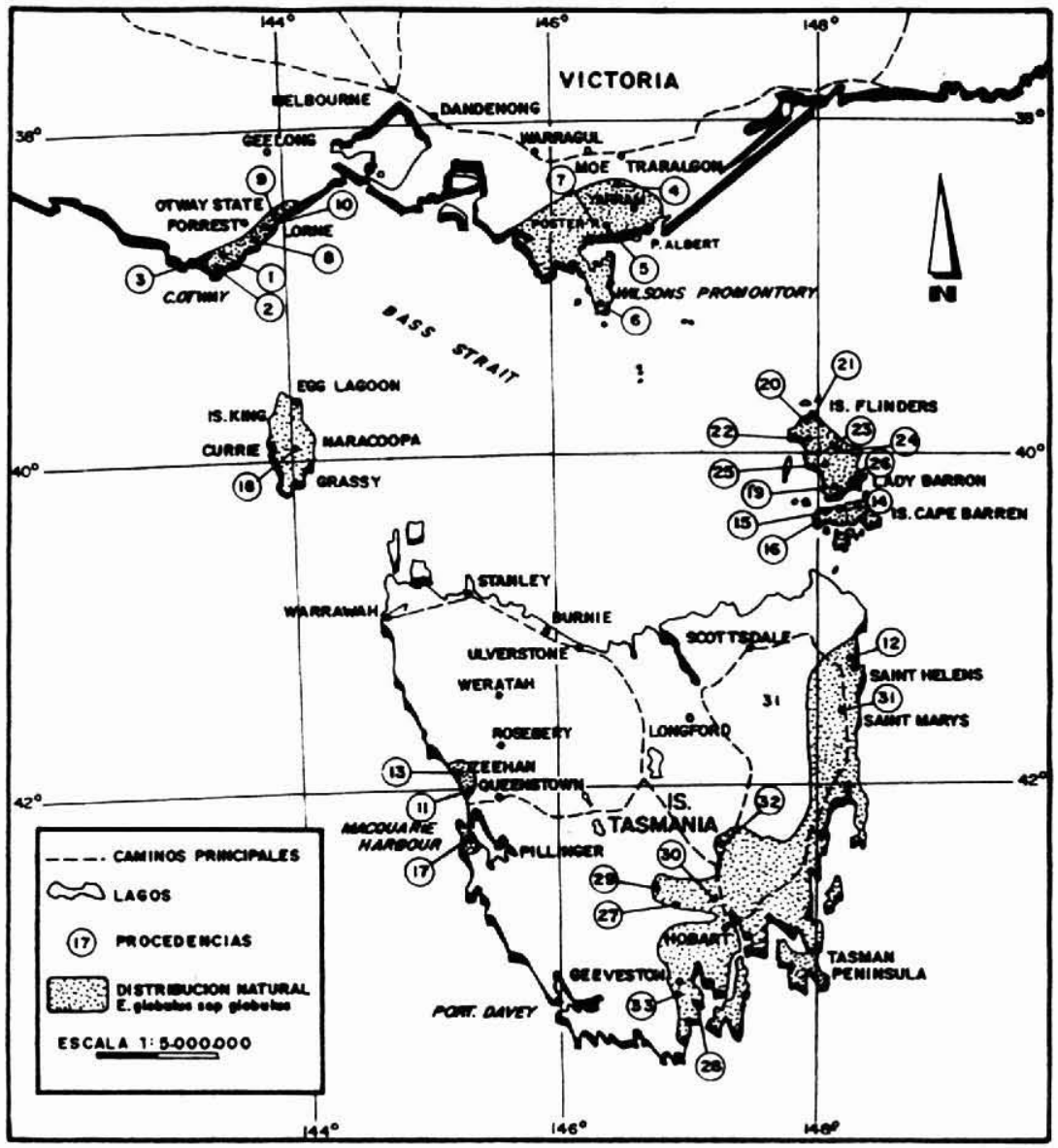

Figura N ${ }^{\circ}$ 1. DISTRIBUCION NATURAL Y PROCEDENCIAS ENSAYADAS DE Eucalyptus globulus ssp. globulus 
Cuadro № 1.

PROCEDENCIAS DE Eucalyptus globulus ssp. globulus ENSAYADAS EN ARAUCO - CHILE

\begin{tabular}{|c|c|c|c|c|c|c|c|c|c|c|c|c|}
\hline \multirow[t]{2}{*}{$\mathrm{N}^{0}$} & \multirow{2}{*}{$\begin{array}{l}\text { Cod. } \\
\text { CSIRO }\end{array}$} & \multirow{2}{*}{\multicolumn{2}{|c|}{ Lugar de Origen }} & \multirow{2}{*}{$\begin{array}{l}N^{\circ} \\
(1)\end{array}$} & \multirow[t]{2}{*}{ Lat. } & \multirow[t]{2}{*}{ Long. } & \multirow[t]{2}{*}{ Alt } & \multicolumn{3}{|c|}{ Precipitación } & \multicolumn{2}{|c|}{ Temp. } \\
\hline & & & & & & & & $\begin{array}{l}(2) \\
(\%)\end{array}$ & $\begin{array}{l}(3) \\
(\%)\end{array}$ & $\begin{array}{r}\text { Total } \\
(\mathrm{mm})\end{array}$ & $\begin{array}{l}(4) \\
\left({ }^{\circ} \mathrm{C}\right)\end{array}$ & $\begin{array}{l}(5) \\
\left({ }^{\circ} \mathrm{C}\right)\end{array}$ \\
\hline 1 & 16223 & Calder Otway & VIC & 4 & $38^{\circ} 46^{\prime}$ & $143^{\circ} 32^{\prime}$ & 200 & 37 & 63 & 870 & 17 & 10 \\
\hline 2 & 224 & Parker Rd Otway & VIC & 7 & $38^{\circ} 49^{\prime}$ & $143^{\circ} 34^{\prime}$ & 145 & 37 & 63 & 870 & 17 & 10 \\
\hline 3 & 16240 & Otway State Forest & VIC & 15 & $38^{\circ} 25^{\prime}$ & $143^{\circ} 27^{\prime}$ & 150 & 37 & 63 & 870 & 17 & 10 \\
\hline 4 & 16319 & Jerralang North & VIC & 30 & $38^{\circ} 19^{\prime}$ & $146^{\circ} 36^{\prime}$ & 220 & 40 & 60 & 1388 & 17 & 6 \\
\hline 5 & 16398 & Hogson Hedley & VIC & 3 & $38^{\circ} 38^{\prime}$ & $146^{\circ} 30^{\prime}$ & 20 & 39 & 61 & 607 & - & - \\
\hline 6 & 16399 & Wilson Promontory & VIC & 9 & $39^{\circ} 08^{\prime}$ & $146^{\circ} 25^{\prime}$ & 60 & - & - & - & - & - \\
\hline 7 & 16400 & Longstaffs Toora & VIC & 2 & $38^{\circ} 37^{\prime}$ & $146^{\circ} 21^{\prime}$ & 180 & 39 & 61 & 607 & - & - \\
\hline 8 & 16402 & nnett River & VIC & 6 & $38^{\circ} 39^{\prime}$ & $143^{\circ} 48^{\prime}$ & 250 & 36 & 64 & .928 & 18 & 9 \\
\hline 9 & 16406 & NW. of Lorne & VIC & 6 & $38^{\circ} 31$ & $143^{\circ} 57^{\prime}$ & 210 & 36 & 64 & 928 & 18 & 9 \\
\hline 10 & 16407 & Henderson Lorne & VIC & 9 & $38^{\circ} 31^{\prime}$ & $143^{\circ} 56^{\prime}$ & 210 & 36 & 64 & 928 & 18 & 9 \\
\hline 11 & 16410 & Badgers Creek & TAS & 7 & $41^{\circ} 59^{\prime}$ & $145^{\circ} 18^{\prime}$ & 120 & 40 & 60 & 2460 & 16 & 6 \\
\hline 12 & 16411 & alaiong Bay & TAS & 5 & $41^{\circ} 16^{\prime}$ & 18 & 120 & 46 & 64 & 788 & 18 & 7 \\
\hline 13 & 16412 & Little Henty River & TAS & 6 & $41^{\circ} 56^{\prime}$ & $145^{\circ} 12^{\prime}$ & 10 & 40 & 60 & 2460 & 16 & 6 \\
\hline 14 & 16417 & N. of Cape Barren Is. & TAS & 5 & $40^{\circ} 22^{\prime}$ & $148^{\circ} 13^{\prime}$ & 20 & 42 & 58 & 785 & 18 & 9 \\
\hline 15 & 16419 & NW. of Cape Barren Is. & TAS & 7 & $40^{\circ} 21^{\prime}$ & $148^{\circ} 07^{\prime}$ & 20 & 42 & 58 & 785 & 18 & 9 \\
\hline 16 & 16421 & 1. of Cape Barren Is. & TAS & 4 & $40^{\circ} 26^{\prime}$ & $148^{\circ} 03^{\prime}$ & 40 & 42 & 58 & 785 & 18 & 9 \\
\hline 17 & 16422 & Macquarie Harbour & TAS & 2 & $42^{\circ} 20^{\prime}$ & $145^{\circ} 20^{\prime}$ & 20 & 42 & 58 & 2527 & - & - \\
\hline 18 & 16424 & King Island & TAS & 4 & $40^{\circ} 00^{\prime}$ & $144^{\circ} 00^{\prime}$ & 60 & 34 & 66 & 901 & 17 & 10 \\
\hline 19 & 16425 & S. of Flinders is & TAS & 3 & $40^{\circ} 14^{\prime}$ & $148^{\circ} 08^{\prime}$ & 120 & 42 & 58 & 785 & 18 & 9 \\
\hline 21 & 16427 & Flinders is & TAS & 3 & $39^{\circ} 45^{\prime}$ & $147^{\circ} 57^{\prime}$ & 40 & 42 & 58 & 763 & 18 & 9 \\
\hline 22 & 16429 & I Flinders Is & TAS & 5 & $39^{\circ} 55^{\prime}$ & $147^{\circ} 57^{\prime}$ & 40 & 42 & 58 & 763 & 18 & 9 \\
\hline 23 & 16431 & Is. & TAS & 8 & $40^{\circ} 02^{\prime}$ & $148^{\circ} 01^{\prime}$ & 190 & 31 & 69 & 736 & - & - \\
\hline 25 & 16433 & ders is. & TAS & 3 & $40^{\circ} 04$ & $148^{\circ} 04^{\prime}$ & 200 & 31 & 69 & 736 & - & - \\
\hline 26 & 16434 & of Flinders Is. & TAS & 3 & $40^{\circ} 16^{\prime}$ & $148^{\circ} 10^{\prime}$ & 40 & 42 & 58 & 785 & 18 & 9 \\
\hline 27 & 16470 & gara & TAS & 16 & $42^{\circ} 47^{\prime}$ & $146^{\circ} 55^{\prime}$ & 500 & 43 & 57 & 1031 & 13 & 5 \\
\hline 28 & 16471 & & TAS & 3 & $43^{\circ} 16^{\prime}$ & $146^{\circ} 59^{\prime}$ & 190 & 39 & 61 & 878 & 17 & 6 \\
\hline 29 & 16472 & $\mathrm{E}$ & TAS & 5 & $42^{\circ} 38^{\prime}$ & $146^{\circ} 82^{\prime}$ & 460 & 43 & 57 & 1031 & 13 & 5 \\
\hline 30 & 16473 & Nortolk & TAS & 3 & $42^{\circ} 43^{\prime}$ & $147^{\circ} 09^{\prime}$ & 300 & 52 & 48 & 790 & 7 & 1 \\
\hline 31 & 16474 & Marys & TAS & 5 & $41^{\circ} 34^{\prime}$ & $148^{\circ} 12^{\prime}$ & 400 & 52 & 48 & 716 & 18 & 9 \\
\hline 32 & 16475 & & TAS & 5 & $42^{\circ} 25^{\prime}$ & $147^{\circ} 16^{\prime}$ & 500 & 49 & 51 & 567 & 16 & 5 \\
\hline 33 & 16476 & f Gee & TAS & 7 & $43^{\circ} 12$ & $146^{\circ} 54^{\prime}$ & 250 & 39 & 61 & 878 & 17 & 6 \\
\hline 35 & 16478 & Tasman Peninsula & TAS & 2 & $43^{\circ} 04^{\prime}$ & $147^{\circ} 50^{\prime}$ & 20 & 46 & 54 & 774 & - & - \\
\hline 36 & & Talca VII Regió & CHILE & & $35^{\circ} 26^{\prime}$ & $71^{\circ} 04^{\prime}$ & & 13 & 87 & 735 & 23 & 8 \\
\hline 38 & & Huerto APPM & TAS & & $41^{\circ} 20^{\prime}$ & $145^{\circ} 10^{\prime}$ & & 86 & 64 & 920 & 17 & 9 \\
\hline 39 & 12653 & E. glob. ssp bicostata & & & & & & & & & & \\
\hline & & S. Traralgon & VIC & & & $146^{\circ} 31$ & 290 & 41 & 59 & 915 & 18 & 9 \\
\hline 40 & & $\begin{array}{l}\text { E. glob. ssp maidenil } \\
\text { South Coast }\end{array}$ & NSW & & $36^{\circ} 13$ & $150^{\circ} 09^{\prime}$ & 400 & 55 & 45 & 944 & 20 & 11 \\
\hline & & $\begin{array}{l}\text { Predio Los Hermano } \\
\text { Costa VIII Región }\end{array}$ & CHILE & & $37^{\circ} 41^{\prime}$ & $73^{\circ} 66^{\prime}$ & 100 & 21 & 79 & 1360 & 19 & 7 \\
\hline
\end{tabular}

(1): Número de árboles madres o familias en la procedencia

(2): Precipitación media anual para el periodo Noviembre - Abril (\%)

(3): Precipitación media anual para el periodo Mayo - Octubre (\%)

(4): Temperatura media máxima anual $\left({ }^{\circ} \mathrm{C}\right)$

(5): Temperatura media minima anual $\left({ }^{\circ} \mathrm{C}\right)$ 
La selección de los árboles y la colecta de semilla fue realizada por CSIRO, en los años 1987 y 1988, siendo ésta parte de la colección "1988 Seed Colections of Eucalyptus globulus ssp. globulus for Tree Improvement Purposes" (Gardiner y Granford, 1988). Entre los criterios considerados en la selección de los árboles se encuentran: tamaño y vigor del árbol; forma del fuste; tipo de copa y tipo de ramas. Junto a esto se reunió información de sitio, que incluye ubicación geográfica (latitud, longitud y altitud), exposición, pendiente, y algunas características del suelo, como textura, color y $\mathrm{pH}$.

\section{Diseño experimental}

El diseño experimental empleado es conocido como bloques de familias compactas o de parcelas divididas, en donde la parcela principal es la procedencia. Dentro de cada procedencia se distribuyen al azar las familias en subparcelas de 4 plantas en línea. Cada procedencia cuenta con 10 repeticiones, constituyendo igual número de bloques. Cada bloque se encuentra rodeado con dos hileras de aislación.

\section{Establecimiento}

El ensayo se estableció a fines de Agosto de 1989 en el predio Los Hermanos, de propiedad de Bosques Arauco, a pocos kilómetros de Antihuala, en la zona costera de la VIII Región del país ( $37^{\circ} 41^{\prime}$ LS y $73^{\circ} 66^{\prime}$ LW).

El sitio presenta una topografía plana a levemente inclinada en algunos sectores. Anterior a la plantación, el suelo tuvo uso agrícola, empleandose en el cultivo de cereales y praderas.

La producción de plantas se llevó a cabo en Santiago, en el vivero que para estos fines dispone INFOR. Se emplearon bolsas plásticas de $500 \mathrm{~cm}^{3}$ de volumen de sustrato. Las plantas fueron marcadas con etiquetas de aluminio con el objeto de mantener la identificación de las familias durante todo el proceso de establecimiento.

Tres meses antes de la plantación se efectuó la preparación del suelo empleando un tractor agricola. Primero se roturó el suelo con subsolador sobre las hileras de plantación ( $60 \mathrm{~cm}$ de profundidad) y posteriormente se laboreó toda la superficie con arado de disco. Debido al uso anterior de la tierra y a la maquinaria empleada, la preparación del sitio resultó muy homogénea. 
Una vez plantado el ensayo se aplicó fertilizante a cada planta, en una mezcla de NPK (Urea $80 \mathrm{~g}$, Superfosfato Triple $50 \mathrm{~g}$ y Sulfato de Potasio $15 \mathrm{~g}$ ). El control de maleza se realizó durante los dos primeros años de establecimiento de la plantación, manteniendo las plantas libres de competencia, principalmente de vegetación herbácea de caracter anual.

\section{Mediciones}

El ensayo ha sido medido en tres ocasiones; en Septiembre de 1989, inmediatamente después de la plantación; en Abril de 1990 y en Abril de 1991. En cada medición se registró sobrevivencia, altura total, diámetro del cuello y estado de las plantas.

\section{Análisis Estadistico}

Con el objeto de comprobar la presencia de diferencias en el desarrollo de las plantas según su lugar de origen y árbol madre, se sometieron los datos a análisis de varianza y al test de comparaciones múltiples de Tukey. El análisis se realizó tanto a nivel de procedencias como de familias. Se emplearon para esto los datos tomados durante el tercer control del ensayo, realizado 20 meses despues del establecimiento.

\section{RESULTADOS Y DISCUSION}

\section{Sobrevivencia}

La sobrevivencia de las plantas en ensayo es alta (96\%) debido a las condiciones ambientales favorables para la especie y a la intensiva silvicultura aplicada en el establecimiento, especialmente al efectivo control de la competencia. Sólo las procedencias $\mathrm{N}^{\circ \mathrm{S}} 26$ y 5 , situadas en Flinders Island y Hogson, respectivamente, presentan una sobrevivencia interior a un $80 \%$ (Cuadro $\mathrm{N}^{\circ} 2$ ). 


\section{Crecimiento}

Para evaluar el crecimiento de las plantas se utilizaron las variables altura total $(H)$, diámetro de cuello (D) y el índice $D^{2} H$, que combina las anteriores y es un buen estimador del crecimiento en volumen.

Los resultados del análisis de varianza indican que existen diferencias estadísticas significativas (para un nivel de confianza de 0.01 ) en el crecimiento de las plantas según la procedencia de las semillas, para las tres variables analizadas.

Las mejores procedencias, a la edad de 20 meses, corresponden a: Central Flinders Island ( $N^{\circ} 25$ y 23), Calder Otway ( $\left.N^{\circ} 1\right)$, Lorne ( $\left.N^{\circ} 9\right)$, Geeveston ( $\left.N^{\circ} 33\right)$ y Cape Barren Island $\left(\mathrm{N}^{\circ} 15\right)$. Por otra parte, las procedencias de menor crecimiento resultaron ser: Wilson Promontory ( $\left.\mathrm{N}^{\circ} 6\right)$ ), Hogson ( $\left.\mathrm{N}^{\circ} 5\right)$, Macquarie Harbour $\left(N^{\circ} 17\right)$ y Saint Marys ( $\left.N^{\circ} 31\right)$ (Cuadro $\left.N^{\circ} 2\right)$.

Al comparar los valores extremos se observa que las plantas provenientes de la parte central de la Isla Flinders crecieron dos veces mas en altura que las plantas de semillas traidas de Wilson Promontory en Victoria.

Sin embargo, aun cuando se advierten diferencias importantes asociadas al lugar de origen de las semillas, no se observa en forma clara un patrón geográfico de variación en el crecimiento de las plantas. Un buen ejemplo de esto se aprecia en el caso de la Isla Flinders donde se observan procedencias de alto desarrollo ( Nos 25 y 23) y, a su vez, procedencias de entre las de más bajo crecimiento (Nos 21 y 6). Estos resultados concuerdan con los obtenidos por Volker y Orme (1988) en ensayos de procedencias de esta especie realizados en Tasmania.

Con el fin de contar con una visión más global del efecto de las fuentes de semillas sobre el crecimiento de los árboles, se agruparon las procedencias por zonas geográficas. Los resultados se entregan en la Cuadro $\mathrm{N}^{\circ} 3$ y corresponden a valores medios ponderados según el número de familias de cada procedencia. Se observa que las mejores fuentes de semillas, despues de 20 meses de instalado el ensayo, corresponden a aquellas del sur-este de Tasmania, en las Islas $\mathrm{Ca}$ pe Barren y Flinders, en el Estrecho de Bass, y en Otway, en Victoria.

No existe correlación entre estos resultados y los obtenidos en etapa de vivero, donde las procedencias de Cape Otway presentaban los mayores desarrollos y las procedencias de Flinders Island los más bajos (Infante y Prado, 1989).

Por otra parte, con el objeto de analizar la variabilidad entre familias se realizaron analisis de varianza (ANDEVA), esta vez al interior de cada procedencia. En la mayor parte de los casos los resultados indican que existen diferencias signifi- 
cativas entre las familias en ensayo. Es asi como la procedencia Lorne $\left(\mathrm{N}^{\circ} 9\right) \mathrm{de}$ Victoria, presenta una variación, para el índice de crecimiento $D^{2} \mathrm{H}$, que va desde $2627 \mathrm{~cm}^{3}$ (familia $\mathrm{N}^{\circ} 84$ ) hasta $4874 \mathrm{~cm}^{3}$ (familia $\mathrm{N}^{\circ} 87$ ). Sin duda que la presencia de esta variabilidad adquiere importancia desde el punto de vista de las ganancias genéticas esperables a partir de la selección de árboles en el ensayo.

\section{Cuadro $\mathrm{N}^{\circ} 2$}

\section{SOBREVIVENCIA, ALTURA, DIAMETRO Y D2H PROMEDIO, A LA EDAD DE 20 MESES, EN ENSAYO DE PROCEDENCIAS DE E. globulus ssp. globulus ARAUCO - CHILE}

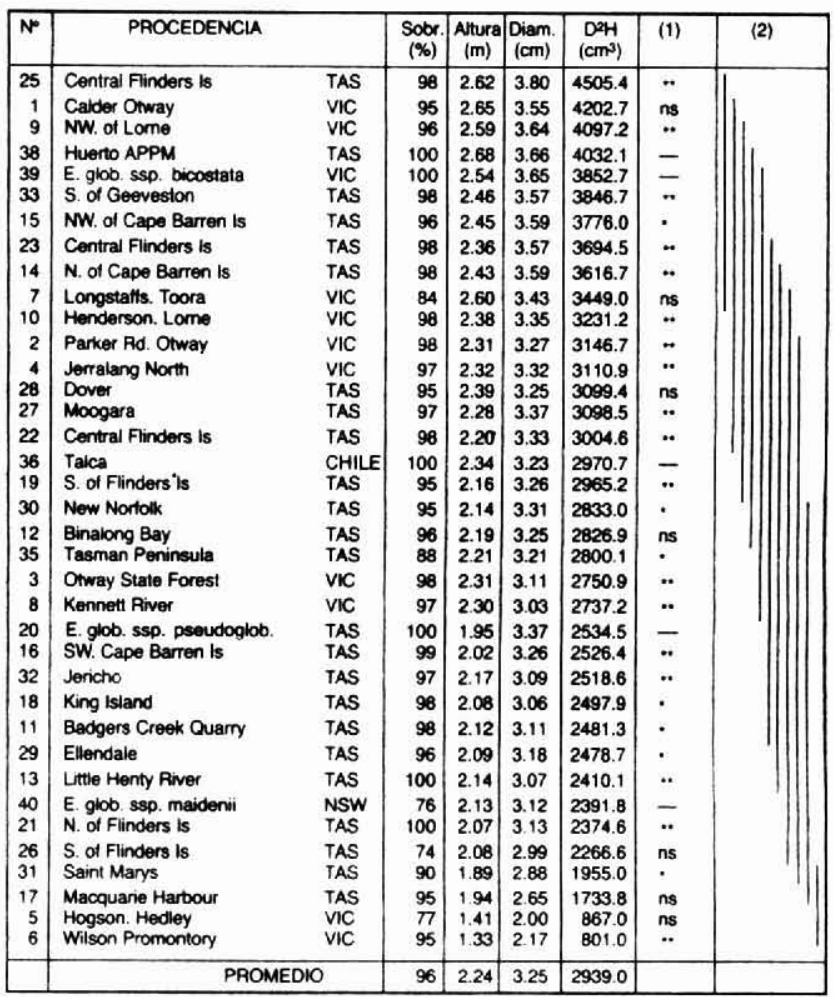

(1) Diferencias estadisticas significativas entre familias dentro de la procedencia. ns No Significativo

- Significativo

* Muy Significativo

(2) Barras indican grupos de medias sin diferencias significativas

Test de Tukey, $\alpha=0.05$ 


\section{Cuadro $\mathrm{N}^{\circ} 3$}

\section{PROCEDENCIAS ENSAYADAS AGRUPADAS POR REGIONES GEOGRAFICAS} E. globulus ssp. globulus. ARAUCO - CHILE EDAD 20 MESES

\begin{tabular}{|ll|c|c|c|c|}
\hline Región Geográfica & & $\begin{array}{c}\mathbf{N}^{\circ} \\
\text { Familias }\end{array}$ & $\begin{array}{c}\text { Altura } \\
(\mathrm{m})\end{array}$ & $\begin{array}{c}\text { Rango } \\
\mathrm{D}^{2} \mathrm{H} \\
\left(\mathrm{cm}^{3}\right)\end{array}$ & $\begin{array}{c}\text { Variación } \\
\left(\mathrm{cm}^{3}\right)\end{array}$ \\
\hline Tasmania Sur-Este & TAS & 10 & 2.5 & 3623 & $3099-3847$ \\
Islas Cape Barren & TAS & 16 & 2.3 & 3413 & $2526-3776$ \\
Lorne Otway & VIC & 21 & 2.4 & 3338 & $2737-4097$ \\
Islas Flinders & TAS & 25 & 2.3 & 3237 & $2266-4505$ \\
Jerralang. Traralgon & VIC & 30 & 2.3 & 3111 & - \\
Cape Otway & VIC & 26 & 2.5 & 3081 & $2751-4203$ \\
Tasmania Central & TAS & 29 & 2.2 & 2864 & $2479-3099$ \\
Península de Tasmania & TAS & 2 & 2.2 & 2800 & - \\
Isla King & TAS & 4 & 2.1 & 2498 & - \\
Tasmania Este & TAS & 10 & 2.1 & 2390 & $1955-2827$ \\
Tasmania Oeste & TAS & 15 & 2.1 & 2353 & $1734-2481$ \\
Yarran & VIC & 5 & 1.9 & 1899 & $867-3449$ \\
Wilson Promontory & VIC & 9 & 1.3 & 801 & - \\
\hline
\end{tabular}

Cuadro $\mathrm{N}^{\circ} 4$

ALTURA, DIAMETRO Y D²H DE LAS MEJORES 25 FAMIULAS DE Eucalyptus globulus ssp. globulus EN ARAUCO-CHILE EDAD 20 MESES

\begin{tabular}{|r|c|c|c|c|ll|}
\hline Familia & $\begin{array}{c}\text { Proc. } \\
\left(\mathrm{N}^{\circ}\right)\end{array}$ & $\begin{array}{c}\text { Altura } \\
(\mathrm{m})\end{array}$ & $\begin{array}{c}\text { Diámetro } \\
(\mathrm{cm})\end{array}$ & $\begin{array}{c}\mathrm{D}^{2} \mathrm{H} \\
\left(\mathrm{cm}^{3}\right)\end{array}$ & Lugar de Origen \\
\hline 162 & 23 & 2.7 & 4,2 & 5574 & Central Flinders & Est. Bass \\
84 & 9 & 2.8 & 3,7 & 5206 & Lorne & Victoria \\
167 & 25 & 2.7 & 4,1 & 5099 & Central Flinders & Est. Bass \\
165 & 25 & 2.7 & 3,9 & 4879 & Central Flinders & Est. Bass \\
90 & 10 & 2,8 & 3,8 & 4843 & Lorne & Victoria \\
216 & 33 & 2.6 & 4,1 & 4745 & Geeveston & Tasmania \\
86 & 9 & 2.8 & 3,8 & 4712 & Lorne & Victoria \\
130 & 15 & 2.7 & 3,9 & 4641 & Capen Barren & Est. Bass \\
3 & 1 & 2,8 & 3,7 & 4631 & Calder Otway & Victoria \\
215 & 33 & 2.7 & 4.0 & 4585 & Geeveston & Tasmania \\
121 & 14 & 2,6 & 4,0 & 4575 & Cape Barren & Est. Bass \\
131 & 15 & 2.6 & 3,8 & 4452 & Cape Barren & Est. Bass \\
214 & 33 & 2,4 & 3.7 & 4433 & Geeveston & Tasmania \\
52 & 4 & 2.7 & 3.8 & 4384 & Jerralang & Victoria \\
96 & 10 & 2,7 & 3,7 & 4327 & Lorne & Victoria \\
160 & 23 & 2.5 & 3.9 & 4295 & Central Flinders & Est. Bass \\
212 & 33 & 2,5 & 3,8 & 4266 & Geeveston & Tasmania \\
150 & 22 & 2,6 & 3.8 & 4254 & Central Flinders & Est. Bass \\
120 & 14 & 2,5 & 3,8 & 4253 & Cape Barren & Est. Bass \\
7 & 2 & 2.5 & 3.7 & 4251 & Parker Otway & Victoria \\
177 & 27 & 2,5 & 3.9 & 4242 & Moogara & Tasmania \\
157 & 23 & 2.5 & 3.7 & 4208 & Central Flinders & Est. Bass \\
2 & 1 & 2,6 & 3,5 & 4205 & Calder Otway & Victoria \\
8 & 2 & 2,6 & 3.6 & 4193 & Parker Otway & Victoria \\
\hline
\end{tabular}


La fuente de semilla nacional, Talca, usada como testigo en el ensayo, se sitúa en una posición intermedia respecto de las procedencias de Australia. Esta situación indica que, si se mantiene esta tendencia en el crecimiento de los árboles, será preferible utilizar semillas de las fuentes australianas de mejor desarrollo en reemplazo de las procedencias locales, en este caso representadas por semilla comercial del Centro de Semillas Chillán. Esto es válido siempre que la semilla local mantenga sus características y no cuente con un mayor grado de mejoramiento. La otra procedencia local, identificada como Cañete, presenta un muy buen desarrollo, pero ha sido excluida del análisis, debido a una condición diferente de las plantas al momento del establecimiento, ya que éstas fueron producidas en macetas con un volumen 5 veces superior a las empleadas para el resto de las plantas.

Desde el punto de vista del mejoramiento genético, los resultados no son argumento suficiente para descartar la raza local como material base de un programa de esta naturaleza. Es importante notar que los valores presentados en el Cuadro $^{\circ} 2$ son valores promedio. Puede ocurrir que la variabilidad de la raza local incluya la variabilidad encontrada en las fuentes naturales. En el caso de ocurrir esto, bastaria con emplear como productores de semillas árboles seleccionados en las plantaciones comerciales existentes en el país. Esto es poco probable, ya que es de suponer que la base genética de las plantaciones locales puede ser bastante restringida.

En la Figura $\mathrm{N}^{\circ} 2$ se muestra la distribución de frecuencias de la altura de los árboles para las procedencias de Talca y Central Flinders ( $\left.N^{\circ} 25\right)$. La curva que representa a Flinders se extiende mas hacia la derecha que la de Talca indicando la presencia de un grupo de árboles superiores en altura, los cuales podrían ser seleccionados, teniendo en cuenta la heredabilidad del caracter, para la producción de semillas, desechando de este modo los de la raza local. Esto mismo se puede observar en la Figura $N^{\circ} 3$ donde se comparan los mejores árboles de las procedencias australianas sobresalientes y de la fuente nacional, Talca.

A pesar de estas diferencias, no es posible afirmar que las procedencias naturales cuentan con un mayor potencial, frente a perspectivas de ganancias genéticas, si se considera que la muestra nacional usada en el ensayo cuenta con pocos individuos y solo representa una procedencia (Talca). Además, el crecimiento de las plantas es solo uno de los atributos a considerar en la selección de árboles. Es común analizar otros caracteres como densidad de la madera, forma del fuste, adaptabilidad y rendimiento pupable, entre otros, con una ponderación que dependerá del producto final a obtener del bosque. 


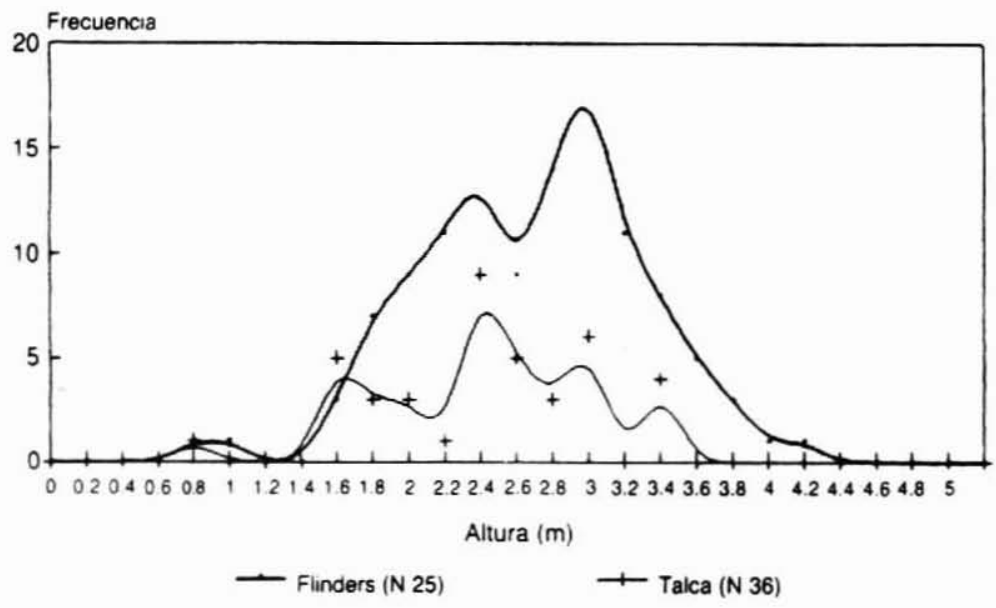

Figura $N^{\circ} 2$. HISTOGRAMA DE FRECUENCIAS PROCEDENCIAS FLINDERS Y TALCA (20 MESES DE EDAD)

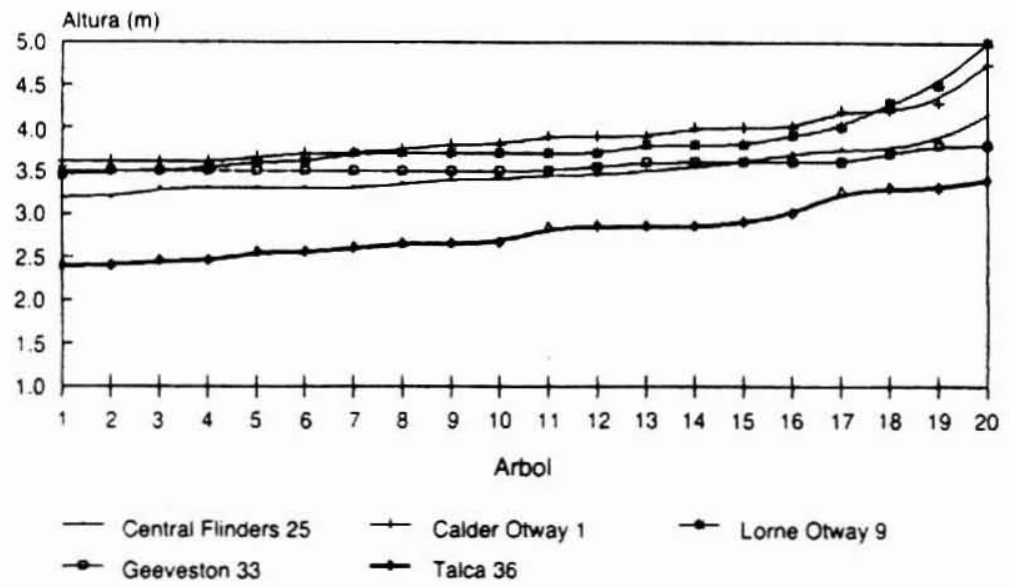

Figura $N^{\circ} 3$. MEJORES 20 ARBOLES de LA PROCEDENCIA TESTIGO Y DE LAS MEJORES PROCEDENCIAS AUSTRALIANAS. (20 MESES DE EDAD) 
La semilla procedente del huerto semillero de APPM ubicado en Tasmania ha demostrado ser de muy buena calidad al situarse entre las procedencias de mejor crecimiento. En este caso se debe considerar que cuenta con un mayor grado de mejoramiento y que representa los resultados obtenidos de ensayos en Australia.

También resulta interesante destacar el crecimiento logrado hasta el momento por E. globulus ssp. bicostata, ubicandose entre las 5 mejores. Plantaciones anteriores con las subespecies de $\mathbf{E}$. globulus ubican a E. globulus ssp. bicostata por debajo de E. globulus ssp. globulus y E. globulus ssp. maidenii (Prado, 1988).

La evaluación de la calidad de la pulpa y su relación con el origen de la semilla es un aspecto de gran importancia a considerar en la futura evaluación del ensayo. Estudios llevados a cabo en Australia indican grandes variaciones en el rendimiento pulpable y en la cantidad de reactivo empleado en el proceso de fabricación de pulpa química (Turner et al., 1983). Los resultados de esta investigación indican que las procedencias ubicadas en la zona Oeste de Tasmania, Henty y Macquarie Harbour, consideradas en este y otros estudios entre las menos apropiadas debido a su bajo crecimiento, son las de mejores características pulpables. En el proceso se utilizó, para la procedencia de Henty, un $50 \%$ menos de reactivo y un $30 \%$ menos de madera en relación a la procedencia de Rheban, para producir una misma cantidad de pulpa. Por otra parte, las procedencias del sur-este de Tasmania, indicadas por algunos autores como la fuente de semillas que dio origen a las plantaciones de E. globulus fuera del territorio australiano, se sitúan en una posición intermedia para estas características.

\section{CONCLUSIONES}

A la edad de 20 meses se observaron importantes variaciones en crecimiento de las plantas según el origen de la semilla. Estas diferencias se encuentran a nivel de procedencia y familia las que, de mantenerse, permitirán obtener altas ganancias genéticas en la futura selección de árboles a partir del ensayo.

Aun cuando no se manifiesta una tendencia geográfica clara, las mejores fuentes de semillas, dado el crecimiento de las plantas, se encontrarian al sur-este de Tasmania, en las islas Flinders y Cape Barren en el Estrecho de Bass y en la zona de Otway en Victoria. 
La semilla utilizada como testigo, procedente de Talca y adquirida en el Centro de Semillas de Chillán, se ubica en una posición intermedia respecto al crecimiento de las plantas. A pesar de ésto, no es posible afirmar, en base a estos resultados, que la raza local es inferior o superior a las fuentes naturales. Para esto será necesario conocer su variabilidad, la que dificilmente esta totalmente comprendida en la muestra empleada en este ensayo. Es probable que en definitiva se utilicen ambas fuentes de semillas en los programas de mejoramiento genético con horizontes a largo plazo.

Al tomar una decisión sobre las fuentes de semillas más apropiadas para el establecimiento de plantaciones con E. globulus, como asimismo en la selección de árboles con fines de mejoramiento genético, no sólo se deben considerar caracteres relacionados con el crecimiento y forma de los árboles, sino que además aparece como de gran importancia tomar en cuenta, entre otros, caracteres asociados a la calidad de la pulpa, cuando este es el objetivo de producción.

\section{RECONOCIMIENTOS}

El Instituto Forestal agradece la colaboración prestada por la Empresa Bosques Arauco S.A. para la realización de esta investigación.

\section{REFERENCIAS BIBLIOGRAFICAS}

Boland, D.J.; M.I. Brooker, G.N. Chippendale; N. Hall, B.P. Hyland, R.D. Johnston, D.A. Kleining e I.D. Turner, 1984. Forest Trees of Australia- Nelson-CSIRO, 687 p.

Gardiner, C.A. y D.F. Granford. 1988. Seed Colections of Eucalyptus globulus ssp. globulus for Tree Improvement Purposes. Australian Tree Seed Centre. CSIRO. Canberra. Australia.

Infante P. y J.A. Prado, 1989. Crecimiento de 35 procedencias de Eucalyptus globulus ssp. globulus en la etapa de vivero. Ciencia e Investigación Forestal № 7: 83 - 89.

Kirkpatrick, J.B., 1975. Geographical variations in Eucalyptus globulus - Forestry and Timber Bureau. Bulletin 47. Canberra-Australia $64 \mathrm{p}$. 
Prado, J.A. 1988., Selección de Procedencias de varias especies del género Eucaiyptus para la zona centro-sur de Chile. En: Actas Simposio manejo Silvicola del Género Eucalyptus. Corporación de Fomento de la Producción. Instituto Forestal, Viña del Mar. Chile, Junio $1988,32 \mathrm{p}$.

Turner, C.H; V. Baladis y G.H. Deam, 1983. Variability in pulping quality of Eucalyptus globulus from Tasmania provenances. Appita. Vol 36 № 5: 371- 376.

Volker P. and Orme, 1988 - Provenance trials of Eucalyptus globulus and related species in Tasmania. Australian Forestry, 51 (4): $257-265$. 